\title{
422. Die Durchblutung der Knochen der hinteren Extremität beim Hund und ihre Veränderungen nach Osteosynthesen
}

\author{
K. Kunze, L. Faupel und W. Völkel \\ Klinik für Unfallchirurgie des Zentrums für Chirurgie der Justus-Liebig-Universität Gießen \\ (Leiter: Prof. Dr. H. Ecke), D-6300 Gießen
}

Blood Supply of Bone of the Hind Leg of the Dog and Haemodynamic Changes After Ostheosynthesis

\begin{abstract}
Summary. The "tracer microspheres" method is suitable for measurement of the blood flow (in $\mathrm{ml} / 100 \mathrm{~g}$ tissue $\times \min$ ) even in the "organ" bone with its small blood flow. The blood supply to cancellated bone is eight times that to cortical bone. The more centrally located bones have a significantly higher blood flow than the peripheral bones. Drilling of the medullary cavity decreases the blood flow significantly more than removal of the periosteum from the diaphysis. Both medullary nailing and osteosynthesis with a plate cause marked increases in the blood supply to the bone operated on.
\end{abstract}

Key words: Tracer microspheres method - Blood supply to bone.

Zusammenfassung. Die „tracer microspheres“-Methode ist geeignet, die Durchblutung auch an dem gering durchbluteten „Organ“-knochen in der Einheit $\mathrm{ml} / 100 \mathrm{~g}$ Gewebe $\times \min \mathrm{zu}$ bestimmen. Die Spongiosa ist ca. 8fach stärker durchblutet als die benachbarte Corticalis und die mehr zentral gelegenen Knochen sind signifikant stärker durchblutet als die weiter peripher gelegenen Knochen. Das Ausbohren der Markhöhle beeinträchtigt die Durchblutung der Corticalis signifikant stärker als die Deperiostierung des Schaftes. Sowohl nach der Marknagelung als auch nach der Plattenosteosynthese kommt es zu erheblichen Steigerungen der Durchblutungswerte im Knochen.

Schliusselwörter: Tracer microspheres-Methode - Durchblutung von Knochen.

\section{Die kältekonservierte allogene Spongiosa bei der Reimplantation von Hüftgelenkstotalendoprothesen}

\author{
R. Ascherl, K. Geißdörfer, F. Lechner ${ }^{1}$ und G. Blümel \\ Institut für Experimentelle Chirurgie (Dir.: Prof. Dr. G. Blümel) der Technischen Universität München, \\ Ismaninger Straße 22, D-8000 München 80 und \\ ${ }^{1}$ Kreiskrankenhaus Garmisch-Partenkirchen ( ̈̈rztl. Dir.: Prof. Dr. F. Lechner)
}

\section{Frozen Allogeneic Cancellated Bone in Reimplantation of Total Hip Replacement}

\begin{abstract}
Summary. The still increasing number of implantations of artificial joints also involves an increasing number of reoperations. In an attempt to bridge osseous defects in the acetabulum and/or in the femur frozen allogeneic cancellated bone $\left(-90^{\circ} \mathrm{C}\right)$ was transplanted in 63 patients. In more than $75 \%$ of these cases good healing was seen at radiological follow-up. Compound implants with cement and metal were not needed. In the micromorphological investigations the tissue proved to be non vital and the tissue-bound fibrinolytic activity increased.
\end{abstract}

Key words: Total hip replacement - Reoperation - Allogeneic cancellated bone.

Zusammenfassung. Durch die immer noch steigende Zahl an Implantationen von Kunstgelenken ist auch die Häufigkeit der Zweit- und Mehrfachoperationen gestiegen. Zur Überbrückung ausgedehnter Defekte im Bereich des Acetabulums wie auch am Femur wurde an 63 Patienten kältekonservierte $\left(-90^{\circ} \mathrm{C}\right)$ allogene Spongiosa transplantiert. In mehr als $3 / 4$ der Fälle zeigt sich auch radiologisch ein gutes Einheilen des Transplantates; auf Zement-Metall-Verbundkonstruktionen konnte verzichtet werden. Mikromorphologisch zeigt sich das Gewebe avital, die gewebeständige fibrinolytische Aktivität ist erhöht.

Schliisselwörter: Hüfttotalendoprothese - Reoperation - Allogene Spongiosa. 